\title{
ELETROCARDIOGRAFIA EM CÃES ANESTESIADOS COM CETAMINA-S OU CETAMINA
}

\author{
ELECTROCARDIOGRAPHY IN DOGS ANESTHETIZED \\ WITH S-KETAMINE OR KETAMINE
}

\author{
Almir Pereira de Souza ${ }^{1}$ Roberta Carareto ${ }^{2}$ Newton Nunes $^{3}$ \\ Alessandra Valeiro Leite ${ }^{1}$ Danielli Parrilha de Paula ${ }^{1}$
}

RESUMO

A cetamina, fármaco derivado da fenciclidina, há muito é usada na anestesia veterinária, sendo que o seu isômero (+), a cetamina $S$, recém-lançada no mercado, tem tido emprego principalmente na anestesia humana. Em vista disso, objetivouse, com este experimento avaliar comparativamente, as possíveis alterações eletrocardiográficas em cães anestesiados com cetamina $S$ ou cetamina e avaliar as freqüências respiratória e cardíaca, saturação de oxihemoglobina e pressão arterial. Para tanto, foram utilizados 10 cães adultos, machos e fêmeas, sem raça definida e hígidos. Os animais foram distribuídos em dois grupos (G1 e G2) de igual número. Ao G1 administrou-se,por via intramuscular, cetamina $S$ na dose de 20mg/kg, e ao G2, cetamina na mesma dose e via de administração empregados no G1. As observações das variáveis tiveram início imediatamente antes da aplicação dos fármacos $(M 1)$ e a cada 10 minutos $(M 2$ M3 e M4, respectivamente). Os dados numéricos foram submetidos ao teste de Tukey, para análises repetidas no grupo, a fim de verificar significado estatístico ou não entre as médias nos vários momentos, sendo considerado o nível de significância de $5 \%(p<0,05)$. Os resultados apresentados foram elevação da freqüência cardíaca com o uso de ambos os fármacos, discretas alterações na pressão arterial, nenhuma variação da $\mathrm{SpO}_{2}$ e impossibilidade de identificar alterações eletrocardiográficas de importância clínica entre os grupos estudados. Assim, pode-se admitir como hipótese conclusiva que as cetaminas $S$ e racêmica apresentam efeitos similares na condutividade elétrica do miocárdio.

Palavras-chave: eletrocardiograma, dissociativos, anestesia.

\section{SUMMARY}

Ketamine is a drug derived of the phenciclidine and has been used in veterinary anaesthesia for a long time. Its $(+$ isomers, sketamine, was recently introduced in the clinical practice and has been used mainly in human anaesthesia. So, this experiment was performed to evaluate comparatively possible electrocardiographic changes in dogs anesthetized with $\mathrm{S}$ ketamine or ketamine and also evaluate heart rate, respiratory rate, oxihemoglobine saturation and arterial pressure. Ten adult male and female dogs were used in this study. All dogs were healthy and mix breed. The animals were divided in two equal number groups ( $\mathrm{Gl}$ and $\mathrm{G} 2$ ). The $\mathrm{G} 1$ received $20 \mathrm{mg} / \mathrm{kg}$ of $S$ ketamine intra-muscularly and $G 2$ received the same dose of ketamine with the injection protocol. The electrocardiographic changes started immediately before the drug administration (M1) and were repeated every 10 minutes (M2, M3 and M4, respectively). The numeric data were submitted to Tukey test for analysis of the repetitions in the group to verify if the averages were statistically significant or not in the several moments with significant value of $5 \%(p<0.05)$. The presented results were heart rate elevation with both drugs, mild arterial pressure changes could also be observed. No changes in the $\mathrm{SpO}_{2}$ were detected and no electrocardiographic changes with clinic importance could be identified. Consequently it is possible to assume as a conclusive hypotheses that $S$-ketamine and ketamine have similar effects over the miocardium electrical conductivity.

Key words: electrocardiogram, dissociative, anesthesia.

\section{INTRODUÇÃO}

A manutenção da anestesia geral em cães é normalmente feita com o uso de anestésicos inalatórios, mas também, é corriqueiro e necessário o uso de fármacos anestésicos injetáveis para a

\footnotetext{
${ }^{1}$ Pós-graduando em Cirurgia Veterinária, Área de Concentração em Cirurgia Veterinária, Faculdade de Ciências Agrárias e Veterinárias (FCAV), Universidade Estadual Paulista (UNESP), Campus de Jaboticabal, SP.

${ }^{2}$ Residente em Anestesiologia Veterinária, Hospital Veterinário "Gov. Laudo Natel”, FCAV, UNESP.

${ }^{3}$ Professor Assistente, Doutor, Departamento de Clínica e Cirurgia Veterinária, FCAV, UNESP, 14870-000, Jaboticabal - SP.E-mail: newton@fcav.unesp.br. Autor para correspondência.
} 
indução e manutenção da anestesia, durante procedimentos cirúrgicos rápidos ou apenas na contenção dos animais. O uso de anestésicos injetáveis apresenta algumas vantagens frente aos inalatórios tradicionais, pela facilidade de administração, custo reduzido e inexistência de poluição ambiental nos centros cirúrgicos.

Dentre as variadas classes de fármacos anestésicos, destacam-se os agentes dissociativos, sendo a cetamina o mais comumente usado para a anestesia dissociativa em cães. Esses fármacos foram introduzidos na Medicina na década de 60 sendo inicialmente liberados para uso em felinos. A característica ausência de depressão cardiorrespiratória quando do uso da cetamina não é igualada por qualquer outro fármaco disponível (BOOTH, 1992).

A cetamina, um derivado da fenciclidina, há muito é usada na anestesia de pequenos e grandes animais, seja, associada a outros fármacos, como agente de indução à anestesia inalatória, para contenção química de indivíduos, seja para procedimentos cirúrgicos de curta duração. Recémlançada no mercado, a cetamina S, o isômero (+) da cetamina, tem tido emprego em anestesia no homem pela sua capacidade de determinar menores estímulos simpáticos e, conseqüentemente, evitar alterações cardiovasculares indesejáveis.

A molécula de cetamina existe como dois isômeros ópticos. Esta mistura racêmica é normalmente usada na clínica; entretanto, quando administrada em animais domésticos, o isômero (+) induz hipnose mais duradoura, quase duas vezes mais longa que o isômero (-) (LIN, 1996). Por outro lado, o efeito da cetamina (+) é duas vezes menor na diminuição da resistência vascular, quando comparado à forma racêmica (LAURETTI et al., s.d.).

A cetamina é caracterizada por ter estimulação cardiovascular indireta. O fármaco aumenta o débito cardíaco, a pressão aórtica média, a pressão arterial pulmonar, a pressão venosa central e a frequiência cardíaca, exercendo efeito variável sobre a resistência vascular periférica (BOOTH, 1992). A frequiência cardíaca e a pressão arterial aumentam como resultado da estimulação direta do sistema nervoso central (SNC), que leva ao aumento do influxo simpático (LIN, 1996).

Alterações relativas à eletrocardiografia, obtidas em cães submetidos à cetamina, foram investigadas por CLARK et al. (1982), que demonstraram terem estas origem em provável hipóxia do miocárdio. Dados semelhantes foram observados por PEREIRA et al. (1992), quando da associação da cetamina à clorpromazina, sendo que, nesse caso, a hipóxia do músculo cardíaco foi constatada devido à observação de constantes infra e supradesnivelamentos do segmento S-T. No coração de rato isolado perfundido, a cetamina altera a atividade elétrica, prolongando os intervalos QR e QT. Entretanto, não induz maiores alterações no padrão do ECG de primatas não-humanos (BOOTH, 1992).

Objetivou-se, com este trabalho, avaliar, comparativamente, a manifestação de possíveis alterações eletrocardiográficas em cães anestesiados com cetamina ou cetamina S. Complementarmente, estudou-se a presença de variações na fisiologia cardíaca e pulmonar que poderiam vir a relacionarse com as alterações de condução elétrica no miocárdio.

\section{MATERIAL E MÉTODOS}

Foram utilizados 10 cães adultos, machos ou fêmeas, sem raça definida, clinicamente sadios, obtidos junto ao canil do Hospital Veterinário "Governador Laudo Natel", da Faculdade de Ciências Agrárias e Veterinárias - FACV / UNESP, Campus de Jaboticabal, SP. Os animais foram distribuídos, aleatoriamente, em dois grupos (G1 e G2), de igual número, e mantidos em jejum alimentar e hídrico por 12 e 3 horas, respectivamente, para, em seguida, receberem os fármacos.

Aos animais do G1 foi administrado, por via intramuscular (IM), cetamina- $\mathrm{S}^{\mathrm{a}}$ na dose de $20 \mathrm{mg} / \mathrm{kg}$, enquanto que nos animais do G2 foi aplicada cetamina ${ }^{\mathrm{b}}$ na mesma dose e via do G1.

Em ambos os grupos, efetuou-se registro do eletrocardiograma (ECG) em derivação DII com emprego de eletrocardiógrafo computadorizado ${ }^{\mathrm{c}}$, pelo qual foram observados os valores referentes à duração e amplitude da onda P (Ps e PmV), duração do complexo QRS (QRSs), amplitude da onda R $(\mathrm{RmV})$, duração do intervalo entre as ondas $\mathrm{Q}$ e $\mathrm{T}$ (QT), intervalo entre duas ondas $\mathrm{R}$ subseqüentes (RR) e polaridade da onda T. Também foi observada a presença, ou não, de figuras eletrocardiográficas anormais.

Foram mensuradas, ainda, freqüência cardíaca (FC) através do intervalo R-R obtido no eletrocardiógrafo computadorizado; freqüência respiratória (FR), pela contagem dos movimentos torácicos em um minuto; saturação de oxihemoglobina $\left(\mathrm{SpO}_{2}\right)$ e pressões arteriais sistólica, diastólica e média (respectivamente PAS, PAD e PAM), investigadas por leitura direta em monitor multiparamétrico $^{\mathrm{d}}$ não invasivo tipo oscilométrico, sendo que, para a $\mathrm{SpO}_{2}$, o emissor/sensor foi 
adaptado em região corpórea dotada de "grau de transparência" compatível com a sensibilidade do dispositivo (lábios, orelhas, prepúcio, mamas). Para as pressões arteriais, o manguito foi adaptado ao membro torácico direito ou esquerdo, acima da articulação úmero-radio-ulnar.

Os momentos estabelecidos para o registro dessas variáveis foram: M1 (antes da aplicação dos fármacos), M2 (10 minutos após a aplicação dos anestésicos), M3 e M4 (10 e 20 minutos após M2).

A avaliação estatística dos dados foi efetuada por meio do Teste de Tukey $(p<0,05)$, usando-se o Sistema de Análises Estatísticas - SAS.

\section{RESULTADOS E DISCUSSÃO}

As variáveis freqüência respiratória e saturação de oxihemoglobina não diferiram estatisticamente $\quad(p<0,05) \quad($ Tabela 1) e mantiveram-se sempre dentro dos limites considerados fisiológicos para a espécie, em ambos os grupos, o que reforça as citações de ser a cetamina o agente anestésico que menos induz à depressão respiratória (BOOTH, 1992).

As curvas da freqüência cardíaca (FC) nos cães de G1 e G2 mostram elevação do parâmetro, confirmando a ação simpatomimética da cetamina, conforme descrito por MASSONE (1999) e NUNES (1995). Esse fenômeno simpatomimético pode ser explicado pela interferência dos fármacos na recaptação de noradrenalina e outras catecolaminas (LUNDY et al., 1986). Não obstante, tais valores permaneceram sempre dentro dos níveis fisiológicos para a espécie.

A observação dos valores da pressão arterial sistólica (Tabela 1) revela que houve um aumento, considerado significativo pelo teste estatístico, 20 minutos após a administração da cetamina S (M3), diferente do G2, em que se observou uma discreta redução deste parâmetro. Tal achado poderia estar relacionado a um estímulo do sistema nervoso simpático promovido pela cetamina $S$, que acarretaria uma elevação do débito cardíaco e da pressão arterial (LIN, 1996). Todas as demais médias de pressão arterial (PAM e PAD) permaneceram dentro de limites fisiológicos para a espécie. Embora o teste estatístico não tenha tido sensibilidade para captar diferenças entre os momentos estudados, observase que, no grupo G2, houve uma redução das médias da PAM e da PAD após a aplicação dos fármacos (M2), ao passo que no grupo G1 estes tenderam a uma discreta elevação. Tal achado no grupo G1 se assemelha com aquele observado por NUNES (1995) com o uso da cetamina. Provavelmente, a via de administração utilizada no presente trabalho (IM) tenha sido a responsável pela não elevação da pressão arterial, resposta farmacológica já descrita deste derivado da fenciclidina (LIN, 1996; KULL et al., 2000). Outrossim, deve-se ressaltar que em humanos a cetamina $S$ tende a determinar menor diminuição da resistência vascular (LAURETTI et al., s.d.) quando comparada à forma racêmica. Por outro lado, o resultado obtido neste experimento não permite concordar com a informação de que a cetamina $\mathrm{S}$ teria ação simpatomimética menor que a da cetamina

Tabela 1 - Valores médios (x) e desvios padrão (s) das variáveis frequiências cardíaca (FC) e respiratória (FR), saturação de oxihemoglobina $\left(\mathrm{SpO}_{2}\right)$, pressões arteriais sistólica, média e diastólica (PAS, PAM e PAD) obtidas em cães anestesiados com cetamina $\mathrm{S}(\mathrm{G} 1)$ e cetamina $(\mathrm{G} 2)$, ao longo dos momentos (M).

\begin{tabular}{|c|c|c|c|c|c|c|}
\hline VARIÁVEIS & & & M1 & M2 & M3 & M4 \\
\hline \multirow{4}{*}{$\begin{array}{l}\mathrm{FC} \\
\text { (bat./min.) }\end{array}$} & \multirow{3}{*}{ G1 } & $\mathrm{x}$ & $107,2 \mathrm{a}$ & $155,4 b$ & $156,2 b$ & $152,6 b$ \\
\hline & & $\mathrm{s}$ & 17,15 & 28,48 & 37,63 & 26,31 \\
\hline & & $\mathrm{x}$ & $99,6 \mathrm{a}$ & $140 \mathrm{~b}$ & $137,8 b$ & $138,8 b$ \\
\hline & \multirow[t]{2}{*}{ G2 } & $\mathrm{s}$ & 21,24 & 23,53 & 18,01 & 18,04 \\
\hline \multirow{5}{*}{$\begin{array}{l}\text { FR } \\
\text { (mov./min.) }\end{array}$} & & & M1 & M2 & M3 & M4 \\
\hline & \multirow{3}{*}{ G1 } & $\mathrm{x}$ & $32,4 \mathrm{a}$ & $29,2 \mathrm{a}$ & $26 a$ & $34 \mathrm{a}$ \\
\hline & & $\mathrm{s}$ & 20,22 & 6,26 & 9,59 & 10,39 \\
\hline & & $\mathrm{x}$ & $34,8 \mathrm{a}$ & $33,4 a$ & $29 a$ & $40,8 \mathrm{a}$ \\
\hline & \multirow[t]{2}{*}{ G2 } & $\mathrm{s}$ & 16,82 & 31,36 & 21,88 & 31,29 \\
\hline \multirow{5}{*}{$\begin{array}{l}\mathrm{SpO}_{2} \\
(\%)\end{array}$} & & & M1 & M2 & M3 & M4 \\
\hline & \multirow{3}{*}{ G1 } & $\mathrm{x}$ & $97,6 a$ & $97 \mathrm{a}$ & $97,6 \mathrm{a}$ & $97,2 \mathrm{a}$ \\
\hline & & $\mathrm{s}$ & 0,54 & 1 & 0,54 & 0,44 \\
\hline & & $\mathrm{x}$ & $97,4 a$ & $96,4 a$ & $97 \mathrm{a}$ & $97 \mathrm{a}$ \\
\hline & \multirow[t]{2}{*}{$\mathrm{G} 2$} & $\mathrm{~s}$ & 0,89 & 1,51 & 1,22 & 1 \\
\hline \multirow{5}{*}{$\begin{array}{l}\text { PAS } \\
(\mathrm{mmHg})\end{array}$} & & & M1 & M2 & M3 & M4 \\
\hline & \multirow{3}{*}{ G1 } & $\mathrm{x}$ & $131,2 \mathrm{a}$ & $124,6 a$ & $132,8 b$ & $116,4 \mathrm{a}$ \\
\hline & & $\mathrm{s}$ & 9,5 & 21,03 & 18,33 & 15,07 \\
\hline & & $\mathrm{x}$ & $138,8 \mathrm{a}$ & $123,2 \mathrm{a}$ & $114,8 \mathrm{a}$ & $131 \mathrm{a}$ \\
\hline & \multirow[t]{2}{*}{ G2 } & $\mathrm{s}$ & 11 & 17,12 & 10,47 & 15,18 \\
\hline \multirow{5}{*}{$\begin{array}{l}\text { PAM } \\
(\mathrm{mmHg})\end{array}$} & & & M1 & M2 & M3 & M4 \\
\hline & \multirow{3}{*}{ G1 } & $\mathrm{x}$ & $90,6 a$ & $95,2 \mathrm{a}$ & $98 \mathrm{a}$ & $96,2 \mathrm{a}$ \\
\hline & & $\mathrm{s}$ & 6,18 & 14,63 & 16,71 & 15,70 \\
\hline & & $\mathrm{x}$ & $101,6 a$ & $87,8 \mathrm{a}$ & $86,4 a$ & $96,4 a$ \\
\hline & \multirow[t]{2}{*}{$\mathrm{G} 2$} & $\mathrm{~s}$ & 13,81 & 15,86 & 9,91 & 6,22 \\
\hline \multirow{5}{*}{$\begin{array}{l}\text { PAD } \\
(\mathrm{mmHg})\end{array}$} & & & M1 & M2 & M3 & M4 \\
\hline & \multirow{3}{*}{ G1 } & $\mathrm{x}$ & $68 \mathrm{a}$ & $76,4 \mathrm{a}$ & $75,8 \mathrm{a}$ & $82,4 a$ \\
\hline & & $\mathrm{s}$ & 5,61 & 13,27 & 16,11 & 17,88 \\
\hline & & $\mathrm{x}$ & $77,8 \mathrm{a}$ & $65,8 \mathrm{a}$ & $70,2 \mathrm{a}$ & $76,2 \mathrm{a}$ \\
\hline & $\mathrm{G} 2$ & $\mathrm{~s}$ & 17,13 & 12,79 & 14,20 & 1,78 \\
\hline
\end{tabular}

Médias seguidas da mesma letra não apresentam diferenças estatisticamente significativas $(\mathrm{p}<0,05)$ entre si na comparação entre os momentos e entre os grupos. 
racêmica (LABORATÓRIO CRISTÁLIA， s.d.) na espécie canina.

Os valores referentes à Ps (Tabela 2), mostraram que não houve diferenças estatísticas entre os grupos, ao longo dos momentos. O maior valor de referência obtida ao longo dos momentos, em ambos os grupos, se deu 20 minutos após a administração dos fármacos. Esse resultado, apesar de não ter tido qualquer implicação clínica, pode significar um discreto retardo da condução elétrica atrial, pois, nos grupos estudados, observou-se aumento das médias de duração da onda $\mathrm{P}$ após a administração de ambas as cetaminas, provavelmente devido a uma elevação da pré e da pós-carga (NUNES et al., 1997), característica desses fármacos.

Com relação à intensidade do impulso elétrico atrial (PmV), não foram encontradas diferenças estatísticas significativas com os anestésicos empregados, estando as médias dentro da faixa de normalidade para a espécie (TILLEY, 1992).

Apesar de não ter tido significado clínico ou estatístico, pode-se observar que a duração dos complexos QRS ficou mais elevada após a aplicação dos anestésicos (Tabela 2), podendo-se verificar, inclusive, que o G1 teve valores inferiores aos do G2, principalmente em M3. Durante todo o período experimental, o tempo de despolarização ventricular manteve-se ligeiramente acima daquele citado por TILLEY (1992) como o normal para a espécie, ou seja, 0,05 segundos. Tal achado, entretanto, não esteve associado com qualquer condição clínica significativa na ocasião. Por outro lado, o método computadorizado de colheita, devido à sua maior precisão, pode mostrar valores ligeiramente diferentes dos considerados fisiológicos, tomados por métodos analógicos.

A amplitude da onda $\mathrm{R}$ (RmV) permaneceu dentro da normalidade para a espécie estudada, não sendo possível detectar diferenças estatísticas entre os grupos pesquisados. Porém, pode-se observar que, no grupo tratado com cetamina $S(\mathrm{G} 1)$, a redução da amplitude desta onda ao longo dos momentos foi mais evidente que aquela registrada no grupo tratado com cetamina (G2). A amplitude da onda R, basicamente, reflete a força contrátil do miocárdio ventricular (TILLEY, 1992). As reduções encontradas neste experimento são condizentes com aqueles registrados por NUNES (1995) quando do uso da cetamina e, provavelmente devem-se a uma alteração da impedância do músculo cardíaco, refletindo um possível aumento da resistência elétrica na musculatura ventricular (SANTOS et al., 2000).
Não houve diferenças estatísticas entre os grupos estudados e nem ao longo dos momentos, para a variável QT (Tabela 2). Todos os valores estão condizentes com os parâmetros de normalidade. O intervalo QT representa a sístole ventricular e é inversamente proporcional à FC (TILLEY, 1992). Tal afirmação fica evidenciada ao se observar que, após a administração dos anestésicos (M2), ocorre uma discreta elevação da frequiência cardíaca que é acompanhada por uma, também discreta, redução do intervalo QT.

No tocante ao segmento RR, pode-se verificar que houve uma redução considerada significativa pelo teste estatístico, após a administração dos fármacos, ao longo dos momentos, não havendo, porém, diferenças entre os grupos. O intervalo R-R representa, em síntese, as

Tabela 2 - Valores médios (x) e desvios padrão (s) de variáveis eletrocardiográficas obtidos em cães anestesiados com cetamina $\mathrm{S}(\mathrm{G} 1)$ e cetamina (G2), ao longo dos momentos (M).

\begin{tabular}{|c|c|c|c|c|c|c|}
\hline Variáveis & & & M1 & M2 & M3 & M4 \\
\hline \multirow[t]{2}{*}{ Ps (mseg) } & G1 & $\begin{array}{l}X \\
S\end{array}$ & $\begin{array}{c}47,4 \mathrm{a} \\
6,58\end{array}$ & $\begin{array}{c}50,6 \text { a } \\
9,55\end{array}$ & $\begin{array}{l}52 \mathrm{a} \\
9,46\end{array}$ & $\begin{array}{c}47,4 \mathrm{a} \\
10,40\end{array}$ \\
\hline & $\mathrm{G} 2$ & $\begin{array}{l}X \\
S\end{array}$ & $\begin{array}{c}42,8 \mathrm{a} \\
4,38\end{array}$ & $\begin{array}{l}48 \mathrm{a} \\
5,83\end{array}$ & $\begin{array}{c}52,8 \mathrm{a} \\
5,44\end{array}$ & $\begin{array}{c}50,2 \mathrm{a} \\
12,11\end{array}$ \\
\hline \multirow{4}{*}{$\mathrm{PmV}(\mathrm{mV})$} & & & M1 & M2 & M3 & M4 \\
\hline & G1 & $\begin{array}{l}X \\
S\end{array}$ & $\begin{array}{c}0,18 \mathrm{a} \\
0,08\end{array}$ & $\begin{array}{c}0,22 \mathrm{a} \\
0,03\end{array}$ & $\begin{array}{c}0,23 \mathrm{a} \\
0,05\end{array}$ & $\begin{array}{c}0,22 \mathrm{a} \\
0,06\end{array}$ \\
\hline & $\mathrm{G} 2$ & $\begin{array}{l}X \\
S\end{array}$ & $\begin{array}{c}0,17 \mathrm{a} \\
0,07\end{array}$ & $\begin{array}{c}0,23 \mathrm{a} \\
0,05\end{array}$ & $\begin{array}{c}0,20 \mathrm{a} \\
0,11\end{array}$ & $\begin{array}{c}0,22 \mathrm{a} \\
0,09\end{array}$ \\
\hline & & & M1 & M2 & M3 & M4 \\
\hline \multirow[t]{2}{*}{ QRS (ms) } & G1 & $\begin{array}{l}\mathrm{X} \\
\mathrm{S}\end{array}$ & $\begin{array}{c}57,2 \mathrm{a} \\
10,91\end{array}$ & $\begin{array}{c}61,4 \mathrm{a} \\
8,90\end{array}$ & $\begin{array}{c}58,6 \text { a } \\
14,46\end{array}$ & $\begin{array}{c}63,4 \mathrm{a} \\
17,09\end{array}$ \\
\hline & $\mathrm{G} 2$ & $\begin{array}{l}X \\
S\end{array}$ & $\begin{array}{c}56,6 \text { a } \\
14,04\end{array}$ & $\begin{array}{l}62,6 \text { a } \\
13,90\end{array}$ & $\begin{array}{c}64,2 \mathrm{a} \\
12,63\end{array}$ & $\begin{array}{c}63 \mathrm{a} \\
14,14\end{array}$ \\
\hline \multirow{4}{*}{$\mathrm{RmV}(\mathrm{mV})$} & & & M1 & M2 & M3 & M4 \\
\hline & G1 & $\begin{array}{l}\mathrm{X} \\
\mathrm{S}\end{array}$ & $\begin{array}{c}1,23 \mathrm{a} \\
0,50\end{array}$ & $\begin{array}{c}1,13 \mathrm{a} \\
0,47\end{array}$ & $\begin{array}{c}1,05 \mathrm{a} \\
0,46\end{array}$ & $\begin{array}{c}1,03 \mathrm{a} \\
0,51\end{array}$ \\
\hline & G2 & $\begin{array}{l}\mathrm{X} \\
\mathrm{S}\end{array}$ & $\begin{array}{c}1,43 \mathrm{a} \\
0,50\end{array}$ & $\begin{array}{c}1,34 \mathrm{a} \\
0,30\end{array}$ & $\begin{array}{c}1,36 \mathrm{a} \\
0,28\end{array}$ & $\begin{array}{c}1,35 \mathrm{a} \\
0,23\end{array}$ \\
\hline & & & M1 & M2 & M3 & M4 \\
\hline \multirow[t]{3}{*}{ QT (mseg) } & G1 & $\begin{array}{l}\mathrm{X} \\
\mathrm{S}\end{array}$ & $\begin{array}{c}206 \mathrm{a} \\
12\end{array}$ & $\begin{array}{l}192 \mathrm{a} \\
18,68\end{array}$ & $\begin{array}{c}189,4 \mathrm{a} \\
21,69\end{array}$ & $\begin{array}{c}187,8 \mathrm{a} \\
42,37\end{array}$ \\
\hline & $\mathrm{G} 2$ & $\begin{array}{l}\mathrm{X} \\
\mathrm{S}\end{array}$ & $\begin{array}{c}197,8 \mathrm{a} \\
2,16\end{array}$ & $\begin{array}{l}194 \mathrm{a} \\
14,45\end{array}$ & $\begin{array}{c}194,6 \mathrm{a} \\
9,20\end{array}$ & $\begin{array}{c}204,8 \mathrm{a} \\
18,71\end{array}$ \\
\hline & & & M1 & M2 & M3 & M4 \\
\hline \multirow[t]{2}{*}{ RR (mseg) } & G1 & $\begin{array}{l}\mathrm{X} \\
\mathrm{S}\end{array}$ & $\begin{array}{c}557 \mathrm{a} \\
262,96\end{array}$ & $\begin{array}{c}349,6 \mathrm{a} \\
103,89\end{array}$ & $\begin{array}{c}366 \mathrm{~b} \\
161,33\end{array}$ & $\begin{array}{c}349,4 \mathrm{~b} \\
91,7\end{array}$ \\
\hline & $\mathrm{G} 2$ & $\begin{array}{l}X \\
S\end{array}$ & $\begin{array}{c}584 \mathrm{a} \\
175,29\end{array}$ & $\begin{array}{c}426,6 \mathrm{~b} \\
70,47\end{array}$ & $\begin{array}{c}453,4 \mathrm{~b} \\
55,73\end{array}$ & $\begin{array}{c}439,2 \mathrm{~b} \\
52,37\end{array}$ \\
\hline
\end{tabular}

Médias seguidas da mesma letra não apresentam diferenças estatisticamente significativas $(p<0,05)$ entre si na comparação entre os momentos e entre os grupos. 
alterações referentes à freqüência cardíaca (TILLEY, 1992). As reduções encontradas neste experimento são condizentes com aqueles registrados por NUNES (1995), o qual, utilizando a cetamina, obteve elevação da FC como conseqüência da ação estimulatória sobre os receptores $\alpha$-adrenérgicos e a inibição parassimpática do coração (SHORT, 1987; MASSONE, 1999). Tal observação também pode ser feita para o presente estudo, em que se verifica aumento da FC após a administração dos fármacos (Tabela 1).

Não foram detectados, ao longo dos momentos, infra ou supradesnivelamento do segmento S-T, não sendo possível caracterizar o achado de hipóxia do miocárdio registrado por PEREIRA et al. (1992). Corrobora tal afirmação o fato de não ter ocorrido, durante o período experimental, depressão da frequiência respiratória e nem da $\mathrm{SpO}_{2}$.

\section{CONCLUSÕES}

Com base nos resultados obtidos com a metodologia empregada, foi possível admitir como hipótese conclusiva que a cetamina $\mathrm{S}$ apresenta efeitos similares àqueles da cetamina racêmica na eletrofisiologia cardíaca e na função cardiopulmonar, não indicando ser este um agente dissociativo de qualidade superior à forma racêmica utilizada rotineiramente.

\section{AGRADECIMENTO}

Os autores agradecem a Fundação de Amparo à Pesquisa do Estado de São Paulo - FAPESP, pelo apoio financeiro concedido, e ao Laboratório Cristália Ltda, pelo fornecimento da cetamina $\mathrm{S}$.

\section{FONTES DE AQUISIÇÃO}

${ }^{a}$ Ketamin S - Cristália Produtos Químicos e Farmacêuticos Ltda. ${ }^{\mathrm{b}}$ Ketamina 50 - Holliday-Scott S.A. - Bs. As. - Argentina

${ }^{\mathrm{c}} \mathrm{Teb}$ - mod. ECGPC software versão 1.10 - Processo FAPESP 96/1151-5

${ }^{d}$ Digimax 5000 - mod. ESFMN 2T - Processo FAPESP 95/2763-1

\section{REFERÊNCIAS BIBLIOGRÁFICAS}

BOOTH, N.H. Anestésicos intravenosos e outros parenterais. In: BOOTH, N. H., McDONALD, L.E. Farmacologia e terapêutica em veterinária. 6 ed. Rio de Janeiro : Guanabara Koogan, 1992. Cap.13, p.168-218.
CLARK, D.M., MARTIN, R.A., SHORT, C.A. Cardiopulmonary responses to xylazine/ketamine anesthesia in dog. J Am Anim Hosp Assoc, v.18, p.815-821, 1982.

KULL, M., KOC, I., ALKAN, F., et al. The effects of xylazineketamine and diazepam-ketamine on arterial blood pressure and blood gases in dogs. Online Journal of Veterinary Research., v.4, n.2, 2000. Disponível em: www.cpd.uokhsc.edu/ojvr. Acesso em: 14 de março. 2000.

LABORATÓRIO CRISTÁLIA. Ketamin S: informações para prescrição. Itapira, s.d. (Folheto Lab.Cristália). np.

LAURETTI, G.R., LIMA, I.C.P.R., BUSCATTI, R.Y., $\boldsymbol{e t}$ al. Avaliação clínica, hemodinâmica, analgésica, psicodélica e anestésica de cetamina racêmica versus seu $S(+)$ isômero. Itapira : Laboratório Cristália, s.d. (Folheto Lab. Cristália). np.

LIN, H.C. Dissociative anesthetics In: THURMON, J.C., TRANQUILLI, W.J., BENSON, G.J. Lumb \& Jones' Veterinary Anesthesia. 3.ed. Baltimore : Lea \& Febiger Book, 1996. p.241-296.

LUNDY, P.M., LOCKWOOD, P.A., THOMPSON, G., et al. Differential effects of ketamine isomers on neuronal and extraneuronal cathecolamine uptake mechanisms. Anesthesiology, v.64, p.359-363, 1986.

MASSONE, F. Anestesiologia veterinária. Rio de Janeiro: Guanabara, 1999. 252p.

NUNES, N. Efeitos cardiocirculatórios da associação de quetamina e levomepromazina, em cães (Canis familiaris) pré-tratados ou não pelo propranolol. Botucatu, 1995. 146p. Tese (Doutorado em Medicina Veterinária) Faculdade de Medicina Veterinária e Zootecnia, Universidade Estadual Paulista, Botucatu, 1995.

NUNES, N., CAMACHO, A.A., COSTA, J.L.O., et al. Eletrocardiographic study of the anesthetic combination of ketamine and chlorpromazine HCL in felines. Braz J Vet Res Anim Sci, v.34, n.1, p.313 - 316, 1997.

PEREIRA, L., LAUS, J.L., NUNES, N., et al. Estudo da associação clorpromazina+quetamina em cães. Braz J Vet Res Anim Sci, v.29, n.2, p.257-265, 1992.

SANTOS, P.S.P., NUNES, N., VICENTI, F.A.P., et al. Estudo eletrocardiográfico, em cães tratados com concentrações decrescentes de desflurano. ARS Veterinaria, Jaboticabal, v.16, n.1, p.16-21, 2000

SHORT, C.E. Principles e practice in veterinary anesthesia. Baltimore : Willian \& Wilkins, 1987. 669p.

TILLEY, L.P. Essential of canine and feline electrocardiography. 3.ed. Philadelphia : Lea \& Febiger, 1992. 470p. 